\title{
THE HAUSDORFF DIMENSION OF SINGULAR SETS OF PROPERLY DISCONTINUOUS GROUPS IN $N$-DIMENSIONAL SPACE
}

BY A. F. BEARDON

Communicated by L. Bers, March 26, 1965

1. Hausdorff dimension. Suppose $E$ is a compact subset of $N$ dimensional euclidean space, $E^{N}$. We denote by $m_{\alpha}(E)$ the Hausdorff $\alpha$-dimensional measure of $E$ and by $d(E)$ the Hausdorff dimension of $E$, i.e. the unique non-negative number such that

$$
m_{\alpha}(E)=0 \quad \text { for } \alpha>d(E)
$$

and

$$
m_{\alpha}(E)=+\infty \quad \text { for } 0 \leqq \alpha<d(E) .
$$

We shall need the following result.

THEOREM A [6]. Let $E$ be a compact subset of $E^{2}$. Then $d(E)>0 \mathrm{im-}$ plies $E$ has positive logarithmic capacity.

2. Spherical Cantor sets.

Definition 1 [2], [7]. We say $E$ is a spherical Cantor set if and only if $E$ can be expressed in the form

$$
E=\bigcap_{n=1}^{\infty} \underset{i_{1}, \cdots, i_{n}=1}{K} \Delta_{i_{1} \cdots i_{n}}
$$

where $K$ is a positive integer $(K \geqq 2)$ and the $\Delta_{i_{1}} \ldots_{i_{n}}$ are closed $N$ dimensional spheres (of radius $r_{i_{1}} \ldots i_{n}$ ) satisfying

(a) $\Delta_{i_{1}} \cdots i_{n} \supset \Delta_{i_{1}} \cdots i_{n+1} \quad\left(i_{n+1}=1, \cdots, K\right)$,

(b) $\Delta_{1}, \cdots, \Delta_{K}$ are mutually disjoint,

(c) there exists a constant $A, 1>A>0$, such that

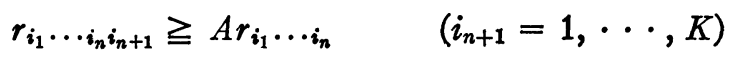

and

(d) there exists a constant $B, 1>B>0$, such that

$$
\rho\left(\Delta_{i_{1} \cdots i_{n} s}, \Delta_{i_{1} \cdots i_{n}}\right) \geqq B r_{i_{1}} \cdots i_{n} \quad(s, t=1, \cdots, K ; s \neq t)
$$

where

$$
\rho(S, T)=\inf \{|s-t| ; s \in S, t \in T\} .
$$

We quote the following results. 
Theorem B [2]. Let $E$ be a spherical Cantor set (in the notation of Definition 1) and suppose that there exist constants $A_{1}, \cdots, A_{\boldsymbol{K}}$ $\left(1>A_{j} \geqq 0\right)$ such that

$$
r_{i_{1} \cdots i_{n} j} \geqq A_{j} r_{i_{1}} \cdots i_{n} \quad(j=1, \cdots, K) .
$$

Then $d(E)$ satisfies

$$
A_{1}^{d(E)}+\cdots+A_{K}^{d(B)} \leqq 1 .
$$

Corollary.

$$
d(E) \geqq-\frac{\log K}{\log A}>0
$$

and so every spherical Cantor set has positive Hausdorf dimension.

THEOREM C [2]. There exists a function $F_{N}(n)$ defined for $n=2,3, \ldots$ such that if $E$ is a spherical Cantor set in $E^{N}$ with $K$ as in Definition 1 then

$$
d(E) \leqq F_{N}(K)<N .
$$

In particular, $d(E)<N$.

3. Inversion groups. For $j=1, \cdots, K+1$ let $S_{j}$ be an $N$-dimensional sphere with centre $a_{j}$ and radius $r_{j}\left(r_{j}>0\right)$. Suppose that $S_{1}, \cdots, S_{K+1}$ are mutually disjoint and for any $x \in E^{N}$ denote by $I_{j}(x)$ the inverse point of $x$ with respect to $S_{j}$. It is well known that $I_{1}, \cdots, I_{K+1}$ generate a properly discontinuous group $G$ with the complement of $U_{j} S_{j}$ as a fundamental region [3], [4]. Let $E$ be the singular set of $G$ and define $E_{j}=E \cap S_{j}$.

THEOREM 1. In the above notation $E_{j}$ is a spherical Cantor set.

Proof. We only sketch the proof here.

Define

$$
\Delta_{i_{1}} \cdots i_{n}=I_{i_{1}} \cdots I_{i_{n-1}}\left(S_{i_{n}}\right), \quad n \geqq 2, i_{t} \neq i_{t+1}
$$

and

$$
\Delta_{i_{1}}=S_{i_{1}}
$$

It is easily seen that

$$
E_{j}=\bigcap_{n=1}^{\infty} \bigcup_{i_{1} \cdots i_{n}-1}^{K+1} \Delta_{i_{1}} \cdots i_{n} \quad\left(i_{1}=j, i_{t} \neq i_{t+1}\right)
$$


and that (apart from relabelling) this is the form required by Definition 1. Further, conditions (a) and (b) of Definition 1 are trivially true. Since we have

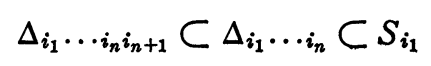

we also have for $k \neq i_{1}$

$$
I_{k}\left(\Delta_{i_{1} \cdots i_{n} i_{n+1}}\right) \subset I_{k}\left(\Delta_{i_{1}} \cdots i_{n}\right) \subset I_{k}\left(S_{i}\right)
$$

and by elementary geometry we can estimate

$$
\frac{\boldsymbol{r}_{k i_{1} \cdots i_{n} i_{n+1}}}{\boldsymbol{r}_{k i_{1} \cdots i_{n}}}
$$

in terms of

$$
\frac{r_{i_{1} \cdots i_{n} i_{n+1}}}{r_{i_{1} \cdots i_{n}}}
$$

In this way we obtain

$$
\frac{r_{k i_{1} \cdots i_{n} i_{n+1}}}{r_{k i_{1} \cdots i_{n}}} \geqq \mu\left(i_{1} \ldots i_{n}\right) \frac{r_{i_{1} \cdots i_{n} i_{n+1}}}{r_{i_{1} \cdots i_{n}}}
$$

where $\mu\left(i_{1} \cdots i_{n}\right)$ depends only upon $S_{1}, \cdots, S_{K+1}$ and $r_{i_{1}} \cdots i_{n}$.

It is easy to show that there exists $\left\{\mu_{n}\right\}$ such that

$$
\mu\left(i_{1} \cdots i_{n}\right) \geqq \mu_{n}>0
$$

and

$$
\prod_{n=1}^{\infty} \mu_{n}=\mu>0
$$

Thus

$$
\frac{r_{i_{1}} \cdots i_{n} i_{n+1}}{r_{i_{1}} \cdots i_{n}} \geqq \mu>0
$$

and so (c) of Definition 1 is verified. The remaining condition (d) can be similarly verified. We remark here that in the case when $r_{1}=\cdots=r_{K+1}=1$ it can be shown that

$$
\frac{r_{i_{1}} \cdots i_{n} i_{n+1}}{r_{i_{1}} \cdots i_{n}} \geqq \frac{1}{\left(f^{2}-1\right) \prod_{n=0}^{\infty}\left(1+\frac{2}{(e-1)^{2 n}}\right)}
$$


where

$$
e=\min \left|a_{p}-a_{q}\right|, \quad f=\max \left|a_{p}-a_{q}\right| \quad(p \neq q) .
$$

A finer estimate in this case gives

$$
\frac{r_{i_{1} \cdots i_{n} i_{n+1}}}{r_{i_{1} \cdots i_{n}}} \geqq \frac{C}{\left|a_{i_{n}}-a_{i_{n+1}}\right|^{2}} \quad\left(i_{n} \neq i_{n+1}\right),
$$

for some positive constant $C$ depending only on $S_{1}, \cdots, S_{K+1}$.

From Theorems B, C and 1 we can immediately deduce the following results.

THEOREM 2. Let $E$ be the singular set of some inversion group. Then $d(E)>0$.

In particular if $r_{1}=\cdots=r_{K+1}=1$ we have

$$
d(E) \geqq \frac{\log K}{2 \log f+\log \prod_{n=0}^{\infty}\left(1+\frac{2}{(e-1)^{2 n}}\right)}>0 .
$$

Theorem 3. There exists a function $F_{N}(p)$ defined for $p=2,3, \ldots$ such that if $E$ is the singular set of any inversion group with $K+1$ generators then

$$
d(E) \leqq F_{N}(K)<N .
$$

We shall indicate a proof of the following result

THEOREM 4. There exists a finitely generated inversion group in $E^{N}$ with singular set $E$ such that $d(E)>N / 2$.

Proof. Let $G_{n}$ be the group uniquely determined by the spheres of unit radius and centres $\left(e_{1}, \cdots, e_{N}\right) \in E^{N}$ where $e_{j}=0,3,6, \cdots, 6 n$ and let $E_{n}$ be the singular set of $G_{n}$. Clearly,

$$
G_{1} \subset G_{2} \subset \cdots
$$

and so

$$
E_{1} \subset E_{2} \subset \cdots
$$

Define

$$
d_{n}=d\left(E_{n}\right)
$$

and

$$
d_{1} \leqq d_{2} \leqq \cdots \leqq d_{n} \leqq \cdots \leqq d=\lim _{n \rightarrow \infty} d_{n} \leqq N
$$


It remains to prove that $d>N / 2$, for then $d_{n}>N / 2$ for some finite value of $n$.

Consider in the construction of $E_{n}$ a fixed sequence $i_{1}, \cdots, i_{p}$. For any $i_{p+1}=1, \cdots,(2 n+1)^{N}$ with $i_{p} \neq i_{p+1}$ the set of numbers

$$
\left|a_{i_{-}}-a_{i_{p+1}}\right|^{2}
$$

always contains the numbers

$$
t_{1}^{2}+\cdots+t_{N}^{2} \quad\left(t_{j}=3,6, \cdots, 3 n\right) .
$$

Thus for the constants $A_{j}$ of Theorem B we can use the $n^{N}$ numbers

$$
\frac{C}{t_{1}^{2}+\cdots+t_{N}^{2}} \quad\left(t_{j}=3,6, \cdots, 3 n\right)
$$

and take the remaining $A_{j}$ 's to be zero. It then follows that

$$
\sum_{t_{1}, \cdots, t_{N}=1}^{\infty} \frac{1}{\left(t_{1}^{2}+\cdots+t_{N}^{2}\right)^{d}}
$$

converges and hence $d>N / 2$ as required.

4. Schottky groups. The definition and details of properly discontinuous groups (and in particular Schottky groups) in the complex plane can be found in [3], [4].

Theorem D [7, p. 109]. Let $G$ be a finitely generated Schottky group in the complex plane with singular set $E$ and suppose that $C_{1}, \cdots, C_{2 p}$ are the circles used in the definition of $G$. Then if $E_{j}$ is that part of $E$ contained in $C_{j}, E_{j}$ is a spherical Cantor set.

ThEOREM E [5]. Let $E$ be the singular set of a finitely generated Schottky group. Then $E$ has positive logarithmic capacity. It follows that the singular set of any properly discontinuous group has positive logarithmic capacity.

From Theorems B, C and D we can deduce the following results.

THEOREM 5. The singular set of any finitely generated Schottky group, and hence of any properly discontinuous group, has positive Hausdorff dimension.

Theorem A implies that Theorem 5 includes Myrberg's wellknown result (Theorem $\mathrm{E}$ ). 
TheOREM 6. There exists a function $F(p)$ defined for $p=2,3, \cdots$ such that if $E$ is the singular set of a Schottky group with $p$ generators then

$$
d(E) \leqq F(p)<2 .
$$

Let $G$ be a Schottky group generated by $T_{1}, \cdots, T_{p}$ such that the mutually external circles $C_{j}, C_{j+p}$ are the isometric circles of $T_{j}, T_{j}^{-1}$ respectively. Suppose further that the radius of $C_{j}$ is $1(j=1, \cdots, 2 p)$. Then $T_{j}$ is equivalent to an inversion in $C_{j}$ followed by a reflection into $C_{j+p}$ (and possibly a rotation). Since it is only the inversion which alters lengths and since the estimates obtained for the inversion groups were uniform over all possible inversions considered, similar theorems can be proved for Schottky groups as for inversion groups, the proofs requiring little modification.

In particular we have

THEOREM 7. There exists a finitely generated Schottky group with singular set $E$ such that $d(E)>1$.

This result has recently been obtained by Akaza [1].

\section{REFERENCES}

1. T. Akaza, Poincare theta series and singular sets of Schottky groups, Nagoya Math. J. 24 (1964), 43-65.

2. A. F. Beardon, On the Hausdorff dimension of general Cantor sets, Proc. Cambridge Philos. Soc. (to be published).

3. L. R. Ford, Automorphic functions, Chelsea, New York, 1929.

4. J. Lehner, Discontinuous groups and automorphic functions, Math. Surveys No. 8, Amer. Math. Soc., Providence, R. I., 1964.

5. P. J. Myrberg, Die Kapazität der singularen Menge der linearen Gruppe, Ann. Acad. Sci. Fennicae Ser. A 10 (1941), 19 pp.

6. S. J. Taylor, On the connexion between Hausdorff measures and generalised capacities, Proc. Cambridge Philos. Soc. 57 (1961), 524-531.

7. M. Tsuji, Potential theory in modern function theory, Maruzen Co., Ltd., Tokyo, 1959, 590 pp.

IMPERIAL COLLEge, LoNdON, ENGLAND AND

UNIVERSITY OF MARYLAND 